\title{
Hsp25 and Hsp70 in rodent tumors treated with doxorubicin and lovastatin
}

\author{
Daniel R. Ciocca, ${ }^{1, *}$ Viviana R. Rozados, ${ }^{2, *}$ F. Darío Cuello Carrión, ${ }^{1}$ Silvia I. Gervasoni, ${ }^{2}$ \\ Pablo Matar, ${ }^{2}$ and 0 . Graciela Scharovsky ${ }^{2}$
}

'Institute of Experimental Medicine and Biology, Regional Center for Scientific and Technological Research, CONICET, c.c. 855, (5500), Mendoza, Argentina

IInstitute of Experimental Genetics, Medical School, National University of Rosario, Rosario, Argentina

\begin{abstract}
Heat shock protein 27 (Hsp27) and Hsp70 have been involved in resistance to anticancer drugs in human breast cancer cells growing in vitro and in vivo. In this study, we examined the expression of Hsp25 (the rodent homologue to human Hsp27) and Hsp70 in 3 different rodent tumors (a mouse breast carcinoma, a rat sarcoma, and a rat lymphoma maintained by subcutaneous passages) treated in vivo with doxorubicin (DOX) and lovastatin (LOV). All tumors showed massive cell death under control untreated conditions, and this massive death increased after cytotoxic drug administration. In this study, we show that this death was due to classic apoptosis. The tumors also showed isolated apoptotic cells between viable tumor cells, and this occurred more significantly in the lymphoma. The tumor type that was more resistant to cell death was the sarcoma, and this was found in sarcomas growing both under control conditions and after cytotoxic drug administration. Moreover, sarcomas showed the highest expression levels of Hsp25 in the viable tumor cells growing under untreated conditions, and these levels increased after DOX and LOV administration. After drug treatment, only sarcoma tumor cells showed a significant increase in Hsp70. In other words, sarcomas were the tumors with lower cell death, displayed a competent Hsp70 and Hsp25 response with nuclear translocation, and had the highest levels of Hsp25. In sarcomas, Hsp25 and Hsp70 were found in viable tumor cells located around the blood vessels, and these areas showed the most resistant tumor cell phenotype after chemotherapy. In addition, Hsp25 expression was found in endothelial cells as unique feature revealed only in lymphomas. In conclusion, our study shows that each tumor type has unique features regarding the expression of Hsp25 and Hsp70 and that these proteins seem to be implicated in drug resistance mainly in sarcomas, making these model systems important to perform more mechanistic studies on the role of Hsps in resistance to certain cytotoxic drugs.
\end{abstract}

\section{INTRODUCTION}

Members of the family of heat shock proteins (Hsps) that are usually present in tumor cells and tissues have been implicated in several key processes in tumor biology. For example, Hsp27 overexpression has been associated with cell growth arrest and increased differentiation in human breast cancer biopsy samples (Vargas-Roig et al 1997). There are 2 responsive elements in the promoter region of the Hsp27 gene, one responding to estrogens or to estrogen receptors and the other to heat shock transcription

Received 9 April 2002; Revised 12 September 2002; Accepted 17 September 2002.

*Contributed equally to this work.

Correspondence to: Daniel R. Ciocca, Tel: 54-261-4286112; Fax: 54-2614287370; E-mail: dciocca@lab.cricyt.edu.ar. factors. Therefore, the Hsp27 response to estrogens can explain the association of this protein with higher cell differentiation not only in breast cancers but also in other estrogen-responsive tumor tissues (Ciocca et al 1989; Puy et al 1989). However, Hsp27 expression can also increase after cell exposure to several stressful conditions (through the heat shock-responsive elements), and for this reason, the evaluation of Hsp27 is not a good marker to predict the responsiveness of human breast cancers to the antiestrogen tamoxifen (Ciocca et al 1998). On the other hand, another member of the Hsp family Hsp70 has been related with higher cell proliferation and with worse prognosis in breast cancer patients (Ciocca et al 1993; VargasRoig et al 1997). Moreover, both Hsp27 and Hsp70 have been involved in resistance to the anticancer drug doxo- 
rubicin (DOX) in human breast cancer cells growing in vitro and in vivo (Ciocca et al 1992; Vargas-Roig et al 1998). At present, this is not surprising because Hsps have protective roles against thermal stress and other damaging conditions. There are several proposed mechanisms to explain the involvement of Hsp27 and Hsp70 in drug resistance (Ciocca and Vargas-Roig 1997; Ciocca and Vargas-Roig 2002); however, so far, the most promising mechanism seems to be the specific requirement of Hsp70 for the survival of tumor cells (depletion of Hsp70 can activate a tumor-specific death program that is independent of caspases and bypasses Bcl-2) (Nylandsted et al 2000). Hsp27 has also been involved in resistance to apoptosis, blocking mitochondrial cytochrome $c$ release (Samali et al 2001).

In a previous study in breast cancer patients, DOX induced significant changes in the expression of Hsp27 and Hsp70, and higher expression levels of these proteins were associated with drug resistance (Vargas-Roig et al 1998). To gain knowledge on the relationship of these Hsps with the response of the tumors to chemotherapy agents, in the present study, we examined the expression of Hsp25 (the rodent homologue to human Hsp27) and Hsp70 in 3 different spontaneous rodent tumors (a mouse breast carcinoma, a rat sarcoma, and a rat lymphoma maintained by subcutaneous [sc] passages) treated in vivo with DOX. In addition, it has been previously shown that lovastatin (LOV) produced a significant inhibition of metastatic growth and invasiveness on a spontaneous rat sarcoma and lymphoma, probably through a decrease in cell adhesion and invasive capacity (Matar et al 1998, 1999). LOV, a drug used in the treatment of patients with hypercholesterolemia, affects the biosynthetic pathway of cholesterol. It competitively inhibits 3-hydroxy-3-methylglutaryl-coenzyme A reductase, blocking mevalonate synthesis, and the generation of farnesyl groups and of nonsterol isoprenoids, thus affecting the posttranslational processing of several proteins (Scharovsky et al 2000). In the current study, LOV was also administered to the animals with tumors, alone and in combination with DOX. In this study, immunohistochemistry was used to examine in detail the expression of Hsp25 and Hsp70 in the tumor cells that survived the cytotoxic drugs and in the cells that were suffering death.

\section{MATERIALS AND METHODS}

\section{Animals}

Inbred adult IIM e/Fm and Fm- $m$ (hereafter referred to as $e$ and $m$, respectively) rats (Calderari et al 1991) from the Facultad de Ciencias Médicas, Universidad Nacional de Rosario breeding facilities, were used. Inbred adult CBi female mice from the Facultad de Ciencias Médicas,
Instituto de Genética Experimental, Universidad Nacional de Rosario breeding facilities, were used (Di Masso et al 1991). Rats and mice were fed with commercial chow and water ad libitum and were maintained in a 12-hour lightdark cycle. All the experiments were developed during the first half of the light cycle. The animals were treated in accordance with the guidelines issued by the Canadian Council on Animal Care.

\section{Drugs}

LOV was kindly provided by Laboratorio SIDUS S.A., Argentina, in the inactive lactone form. It was converted to the active form by dissolving $480 \mathrm{mg}$ of lactone in 12.5 $\mathrm{mL}$ of $96^{\circ}$ ethanol, adding $18 \mathrm{~mL}$ of $0.1 \mathrm{M} \mathrm{NaOH}$, heating at $50^{\circ} \mathrm{C}$ for 2 hours, neutralizing the solution with $0.1 \mathrm{M}$ $\mathrm{HCl}$ to $\mathrm{pH} \mathrm{7,} \mathrm{and} \mathrm{adjusting} \mathrm{with} \mathrm{distilled} \mathrm{water} \mathrm{to} \mathrm{a} \mathrm{vol-}$ ume of $60 \mathrm{~mL}$. Aliquots of this stock solution $(20 \mathrm{mM})$ were stored frozen at $-20^{\circ} \mathrm{C}$. DOX was diluted to $1 \mathrm{mg} /$ $\mathrm{mL}$ in saline solution before injection.

\section{Tumors}

Lymphoma TACB (L-TACB) is a poorly differentiated Bcell lymphoma, which arose spontaneously in an inbred $e$ rat (Celoria et al 1986). It is maintained by serial sc grafting by trocar of $1 \mathrm{~mm}^{3} \mathrm{~L}$-TACB fragments (approximately $10^{6}$ cells) in syngeneic rats. When L-TACB is injected subcutaneously, lymph nodes are the exclusive sites of metastatic growth. Sarcoma E-100 (S-E100) is a fibrosarcoma that appeared spontaneously in an outbred population of IIM rats in 1955, and it is maintained by SC passages in rats of the allogeneic inbred line $m$ with $100 \%$ lethality and $0 \%$ spontaneous metastasis (Doménico et al 1963). Instead, a high incidence of lung metastasis is observed when the same animals are injected intravenously with S-E100 cells. M-406 is a type B semidifferentiated mammary adenocarcinoma (Squartini 1979) that shows a mixed pattern and develops lung and liver metastases. It arose spontaneously in an inbred CBi female mouse. It is maintained in vivo by serial intraperitoneal injections of $2.5 \times 10^{5}$ cells in syngeneic mice. In this location, it is highly invasive to the muscular abdominal wall.

\section{Experimental model}

The experimental procedure followed with the 3 tumor models is as follows. Rats were challenged with L-TACB or S-E100 and mice with M-406 subcutaneously in the right flank, by trocar, on day 0 , and the animals were divided into 4 groups and treated as follows-L-TACB: (1) control animals received no further treatment; (2) injected intraperitoneally with LOV $(25 \mathrm{mg} / \mathrm{kg}$ of body weight) daily, from day 0 to 4 ; (3) injected intraperito- 
neally with DOX $(0.25 \mathrm{mg} / \mathrm{kg})$ on day 10 ; and (4) injected with LOV + DOX as in groups 2 and 3. S-E100: (1) control; (2) injected intraperitoneally with LOV $(25 \mathrm{mg} / \mathrm{kg}$ of body weight) 3 times a week from day 7 to 16 ; (3) injected with DOX (2 $\mathrm{mg} / \mathrm{kg}$ of body weight) twice a week from day 7 to 16; and (4) LOV + DOX as in groups 2 and 3. M-406: (1) control; (2) injected intraperitoneally with LOV $(25 \mathrm{mg} / \mathrm{kg}$ of body weight) 3 times a week from day 5 to 23; (3) injected with DOX ( $1 \mathrm{mg} / \mathrm{kg}$ of body weight) twice a week from day 5 to 23; (4) LOV + DOX as in groups 2 and 3 . The treatment schedules are different because we were testing different combinations of doses and days of administration to achieve, in each tumor model, a maximum antitumor effect with the combination of drugs in nontoxic doses for the animals. The growth of primary tumors was monitored twice a week. All the animals were sacrificed on days 21,18 , and 26 for L-TACB, S-E100, and M-406, respectively, and their tumors resected. The lag time between the end of the drug treatment and the excision of the tumors was different (specially for lymphomas); this was due to one of the criteria followed to decide the end of the experiment: the day on which the control animals reached the maximum permitted tumor size in an in vivo experiment. However, because this relatively long lag time may influence the expression of the Hsps, lymphomas were also taken 3 days after the end of the experiment, which is comparable with the lag time of sarcomas and mammary carcinomas.

\section{Immunohistochemistry}

Tissues were fixed in 10\% buffered formalin and embedded in paraffin. For comparative purposes, the tumors were examined in their largest dimension. Sections of 5 $6 \mu \mathrm{m}$ thickness were immunostained to reveal Hsp25 and Hsp70 using an immunoperoxidase method (Dako Envision System, Dako Corporation, Carpinteria, CA, USA). The antibodies used were (1) rabbit polyclonal antibody against recombinant Hsp25/27, (2) mouse Mab BRM-22 against the constitutive and inducible forms of Hsp70, and (3) rat Mab 1B5 against the constitutive form of Hsp70 (Hsc70), kindly provided by Dr A. Laszlo (Mallinkrodt Institute of Radiology, Washington University Medical Center, St Louis, MO, USA). Detailed description of these antibodies and methods has been published elsewhere (Vargas-Roig et al 1998). In addition, in lymphomas, blood vessels were examined by immunostaining with antibodies against FVIII and FXIIIa using commercial antibodies (Dako Corporation). The immunostained slides were lightly counterstained with hematoxylin and observed and photographed with an IM35 microscope (Carl Zeiss, Oberkochen, Germany). The positive reaction was evaluated considering the location of the specific immunostaining on the tumor cells (nuclear, cytoplasmic), using a semiquantitative grade according to the intensity of immunostaining and the proportion of stained cells (Vargas-Roig et al 1998). Briefly, intensity scores are as follows: no staining, 0 ; weak staining, 1 ; moderate staining, 2; and strong staining, 3. Proportion scores are as follows: $<1 \%, 0 ; 1-10 \%, 1 ; 11-30 \%, 2 ; 31-66 \%$, 3; and $>66 \%$, 4. Immunostained blood vessels were counted under the $40 \times$ objective in the tumor areas showing the highest positive vessels.

\section{TUNEL technique}

Apoptosis was revealed by a modification of the terminal deoxynucleotidyl transferase-mediated dUTP nick end labeling (TUNEL) method, using the ApopTag Plus in situ detection kit (Oncor, Gaithersburg, MD, USA). These modifications have been published elsewhere (CuelloCarrión and Ciocca 1999; Bonfil et al 2001).

\section{Western blot analysis}

Western blot was performed on tissue homogenates prepared as reported previously (Vargas-Roig et al 1997). The protein lysate ( $20 \mu \mathrm{g}$ per lane) was subjected to electrophoresis on $12.5 \%$ sodium dodecyl sulfate-polyacrylamide gels $(\mathrm{w} / \mathrm{v})$ and transferred to nitrocellulose membranes. The membranes were incubated with the antibodies against the Hsps (1:2000), and the bands were developed using chemiluminescence reagents (Dupont NEN, Boston, MA, USA) and autoradiography film (Sigma, St. Louis, MO, USA).

\section{Statistical analyses}

Statistical analyses were performed using the Prism computer program (Graph Pad Software, San Diego, CA, USA). The tests used were the unpaired $t$-test and the Mann-Whitney nonparametric test, with a 2-tailed option and with $95 \%$ confidence interval. A value of $P<0.05$ was considered significant.

\section{RESULTS}

\section{Tumor sizes and cell death}

The tumor sizes of the controls and treated animals, determined on the day of sacrifice, are shown in Table 1 . Although the cytotoxic treatments decreased the tumor sizes, a statistically significant reduction in tumor size was observed only in breast carcinomas after the combined treatment with LOV and DOX. However, tumor areas with massive cell death were observed in the 3 different tumors under study, growing in control conditions and after cytotoxic drug administration. The detailed 
Table 1 Effect of the different treatments on tumor volume

\begin{tabular}{|c|c|c|c|c|c|}
\hline \multirow[b]{2}{*}{ Tumors } & \multirow{2}{*}{$\begin{array}{l}\text { Days after last } \\
\text { drug injection }\end{array}$} & \multicolumn{4}{|c|}{ Tumor volume $\left(\mathrm{cm}^{3}\right)^{\text {a }}$ mean $\pm \mathrm{SE}$} \\
\hline & & Control & DOX & LOV & LOV + DOX \\
\hline Breast carcinoma & 3 & $3.08 \pm 0.62$ & $2.72 \pm 0.35$ & $2.15 \pm 0.39$ & $1.80 \pm 0.24^{b}$ \\
\hline Sarcoma & 2 & $25.38 \pm 4.21$ & $18.85 \pm 1.05$ & $22.15 \pm 4.95$ & $18.09 \pm 1.13$ \\
\hline Lymphomac & 18 & $34.47 \pm 8.28$ & $30.00 \pm 6.76$ & $49.81 \pm 6.86$ & $25.30 \pm 7.86$ \\
\hline Lymphoma $^{d}$ & 3 & $19.03 \pm 3.33$ & $22.15 \pm 2.10$ & $27.74 \pm 13.87$ & $14.34 \pm 9.34$ \\
\hline
\end{tabular}

DOX, doxorubicin; LOV, lovastatin.

a Tumor volume measured at the end of the experiment on the day indicated in the second column.

b Student's $t$-test: control vs LOV + DOX; $P<0.05$.

${ }^{c d}$ Identical experiments but ending 18 and $3 \mathrm{~d}$ after the last injection, respectively.
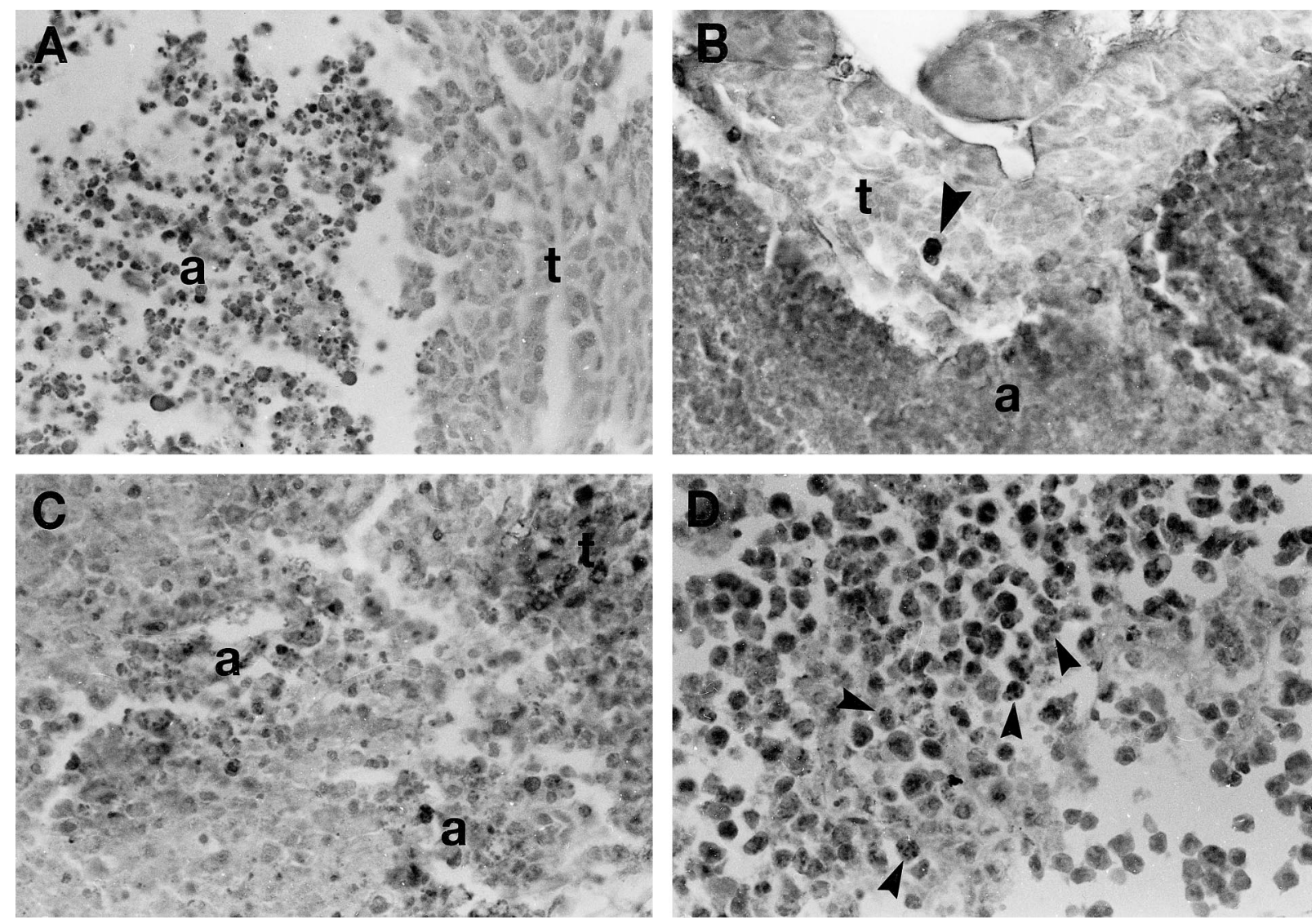

Fig 1. Apoptosis in the 3 tumor types under study. (A) Breast carcinoma growing under control untreated conditions. A large area with massive cell death (a) is seen. Note that the cells display the morphological characteristics of classic apoptosis. t, viable tumor cells. Hematoxylin staining. (B) Breast carcinoma treated with doxorubicin (DOX) and lovastatin. The TUNEL technique stained in dark, both a large area of massive apoptosis (a) and an isolated apoptotic cell (arrowhead in the area of viable tumor cells, $t$ ). TUNEL technique with hematoxylin counterstaining. (C) Sarcoma treated with DOX. There are 2 foci of dead cells (a) displaying the morphological characteristics of classic apoptosis. This tissue section was immunostained to reveal Hsp70 that was absent in apoptotic cells and present in some tumor cells (seen as dark cells in the upper right corner, t). Hematoxylin counterstaining. (D) Lymphoma treated with DOX. The TUNEL technique stained in dark numerous apoptotic cell (arrowheads). TUNEL technique with hematoxylin counterstaining. For comparative purposes, all micrographs are at $120 \times$ magnification.

morphological analysis of these areas revealed that most of the tumor cells were dying by classic apoptosis (Fig 1 $\mathrm{A}, \mathrm{C})$, and very few cells displayed morphological characteristics of apoptosis-like programmed cell death and of necrosis (death types reviewed by Leist and Jäättelä 2001). This finding was confirmed by the in situ TUNEL technique (Fig 1 B,D). The evaluation of the massive apoptotic areas revealed that control untreated tumors showed different degrees of massive apoptosis. Breast carcinomas showed almost a 2-fold increase in massive apoptosis compared with sarcomas, whereas lymphomas showed 1.5-fold increase compared with sarcomas (Fig 

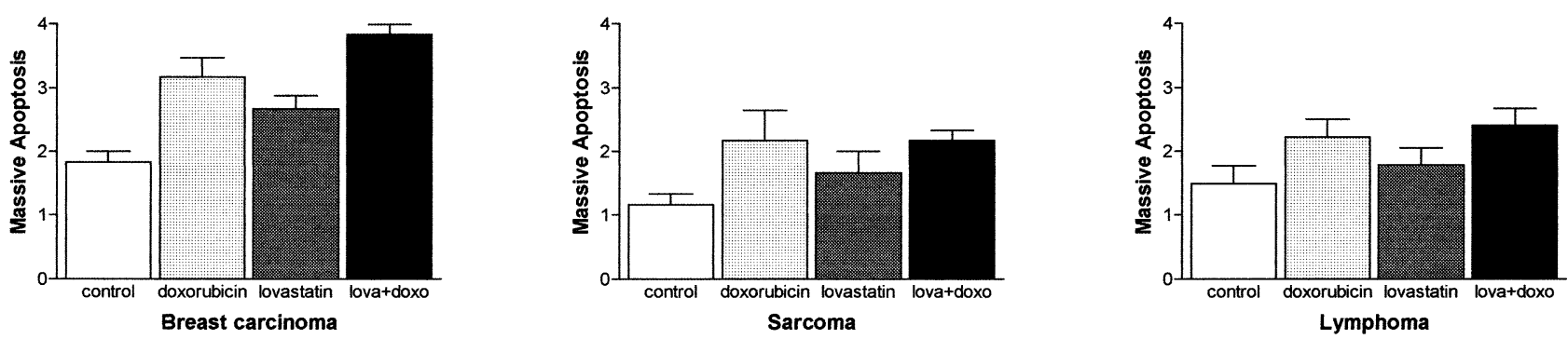

Fig 2. Massive apoptosis in untreated tumors and in tumor treated with doxorubicin and lovastatin. The scores used were: (1) $<10 \%,(2)$ $11-33 \%$, (3) 34-66\%, and (4) $>66 \%$. These scores represent the area (in percentage) showing massive apoptosis, evaluating the entire tumor (examining the tumors in their largest dimension). For instance, there were tumors where $50 \%$ of the tissue section was occupied by massive apoptosis (in this case the score was 3).
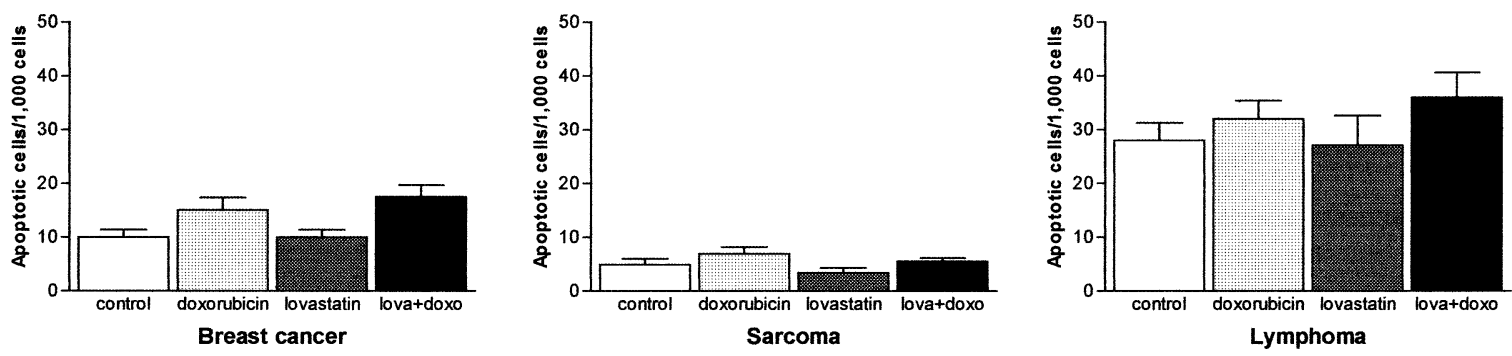

Fig 3. Isolated apoptosis in untreated tumors and in tumors treated with doxorubicin and lovastatin. The scores reflect the number of apoptotic cells per 1000 tumor cells (evaluated in the areas of viable tumor cells).

2). In breast carcinomas, massive apoptotic areas were clearly seen in the center of tumor cell clusters, whereas in sarcomas and lymphomas, areas of massive apoptosis occurred at random without a special arrangement of the viable tumor cells. It was of interest to evaluate the level of massive apoptosis after the different drug treatments. In breast carcinomas, massive apoptosis increased significantly $(P<0.05)$ after DOX treatment and even more in the tumors treated with the combination of LOV and DOX $(P<0.01)$ (Fig 2). LOV alone was also effective in increasing massive apoptosis but at lower level than was DOX alone $(P<0.05)$. In sarcomas and lymphomas, DOX significantly increased massive apoptosis $(P<0.05)$ but at lower levels than in breast carcinomas. In these tumors, LOV increased massive apoptosis but without reaching statistical significance and slightly increased apoptosis when administered in combination with DOX (Fig 2).

Isolated apoptotic cells were also observed intermingled in the viable tumor areas, appearing as single dead cells (Fig 1B) or as small apoptotic cell clusters (Fig 1C). The difference in magnitude of dead cells found as isolated (or small clusters) apoptosis vs massive apoptosis ranged in the order of 1:100 to 1:1000. Although massive apoptosis was significantly higher than isolated apoptosis, the latter might be significant in tumor biology, and for this reason it was also evaluated. Different levels of isolated apoptosis were found in the 3 tumor types studied (Fig 3). Under control conditions, sarcomas showed the lowest level of isolated apoptosis, whereas lympho- mas showed the highest levels. In animals treated with DOX alone or in combination with LOV, breast carcinomas showed more isolated apoptosis than did control tumors $(P<0.05)$ (Fig 3). In sarcomas, the drug treatments did not induce significant changes, and isolated apoptosis remained at very low levels. In contrast, in lymphomas, DOX increased isolated apoptosis and even more when administered in combination with LOV $(P<0.001)$ (Fig 3).

\section{Heat shock protein 25}

In untreated breast carcinomas, viable tumor cells lacked Hsp25 (Fig 4A). In areas of massive apoptosis, the protein was strongly positive, appearing in apoptotic cells (Fig $4 \mathrm{~A})$ and only occasionally in "viable" tumor cells located in the immediate vicinity of apoptotic cells. Most of the isolated apoptotic cells located between the viable tumor cells lacked Hsp25. Western blotting could not discriminate the detailed occurrence of Hsp25 (or Hsp70) in the tumors. We have used the antibodies in Western blot before (Ciocca et al 1989, 1993), but in the present study, for the detailed analysis of the Hsps in the viable tumor cells and in the apoptotic cells, Western blot was not useful, and for this reason we continued this study by immunohistochemistry. In contrast to breast carcinomas, in untreated sarcomas, viable tumor cells expressed Hsp25 (Fig 4B). Table 2 shows the average expression of Hsp25 in the cytoplasm and nucleus of viable tumor cells. Hsp25 

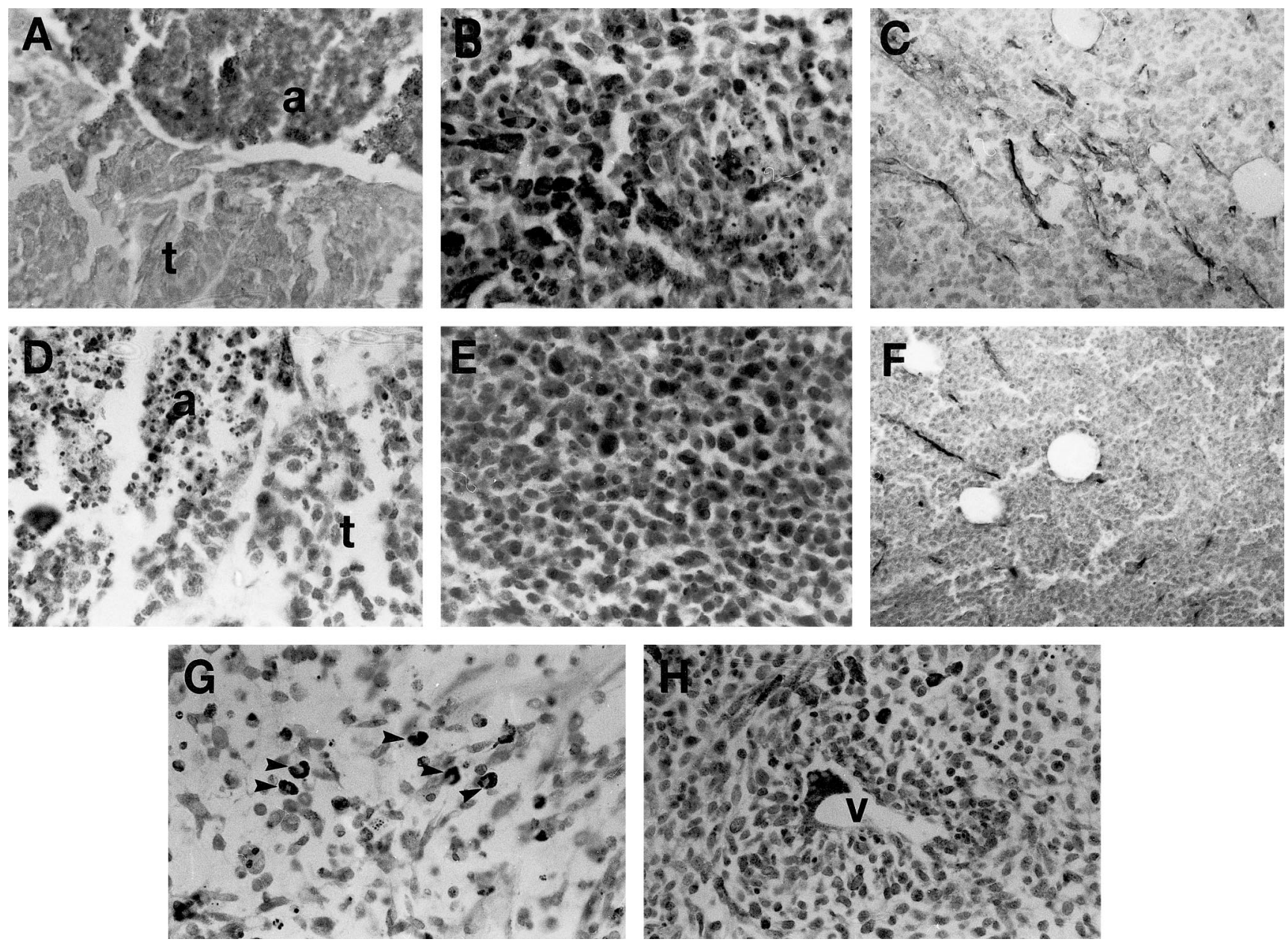

Fig 4. Hsp25 expression in spontaneous rodent tumors (immunostaining for Hsp25 with hematoxylin counterstaining). (A) Breast carcinoma growing under control untreated conditions. The viable tumor cells (t) lacked Hsp25 expression, whereas apoptotic cells (a) showed immunostaining. (B) Tissue section from an untreated sarcoma. Note Hsp25 expression in numerous tumor cells (dark cells) and the presence of some apoptotic cells lacking the protein (right side). (C) Untreated control lymphoma showing numerous small vessels with Hsp25 in endothelial cells. These vessels lacked FVIII immunostaining. (D) In this breast carcinoma treated with doxorubicin (DOX) the viable tumor cells (t) and the apoptotic cells (a) lack Hsp25 expression. (E) Tissue section from a sarcoma treated with DOX. Note that most of the tumor cells express Hsp25. (F) Lymphoma treated with DOX. Hsp25 can be observed in the endothelium of small vessels, but the number of positive vessels decreased when compared with untreated tumors (shown in C). (G) Sarcoma treated with DOX showing cells with strong Hsp25 expression (arrowheads). (H) Sarcoma treated with lovastatin and DOX. The tumor cells located around the blood vessel (V) are the cells that express more Hsp25. For comparative purposes, all micrographs are at 120× magnification except $\mathrm{C}$ and $\mathrm{F}$ that were taken at lower magnification $(60 \times)$.

was absent in areas of massive apoptosis and in isolated apoptotic cells between the viable tumor cells (Fig 4B). Finally, in untreated lymphomas, Hsp25 was absent in viable tumor cells and in tumor cells dying in massive and in isolated apoptoses. It is of interest to mention that the endothelium of the blood vessels located between the tumor cells showed Hsp25 immunostaining (Fig 4C). Western blotting was not sensitive enough to reveal the Hsp25 found in the blood vessels by immunohistochemistry.

In DOX-treated breast carcinomas, Hsp25 could be detected in the cytoplasm of viable tumor cells but with weak staining and in few cells (Table 2). In the areas of massive apoptosis, in contrast to control tumors, Hsp25 was absent in apoptotic cells (Fig 4D). We already mentioned that these massive apoptotic areas were larger than those observed in the control tumors. Moreover, DOX-induced apoptosis appeared as an accelerated phenomenon with lakes and masses of confluent chromatin compaction. Hsp25 expression was weak and remained in very few tumor cells, limiting the massive apoptotic areas. Individual apoptosis between viable tumor cells was Hsp25 negative. In DOX-treated sarcomas, viable tumor cells had expression levels of Hsp25 similar to those observed in the controls (Fig 4E); however, there were areas of viable tumor cells with Hsp25 expression in the nuclei (Table 2). Tumor cells at the borders of massive apoptotic areas adopted elongated appearance, and some 
Table 2 Viable tumor cells, Hsp25 expression in untreated tumors and in tumors treated with doxorubicin (DOX) and lovastatin (LOV)

\begin{tabular}{|c|c|c|c|c|c|c|c|c|}
\hline \multirow[b]{2}{*}{ Tumor } & \multicolumn{2}{|c|}{ Untreated } & \multicolumn{2}{|c|}{ DOX } & \multicolumn{2}{|c|}{ LOV } & \multicolumn{2}{|c|}{ LOV + DOX } \\
\hline & $\mathbf{C}^{\mathrm{a}}$ & $\mathbf{N}$ & C & $\mathbf{N}$ & C & $\mathbf{N}$ & C & $\mathbf{N}$ \\
\hline Breast carcinoma & $0^{\mathrm{b}}$ & 0 & $1+1$ & 0 & $1+1$ & 0 & $1+1$ & 0 \\
\hline Sarcoma & $1+3$ & 0 & $1+2$ & $1+1$ & $1+2$ & $1+1$ & $2+2$ & $1+2$ \\
\hline Lymphoma & 0 & 0 & 0 & 0 & 0 & 0 & 0 & 0 \\
\hline
\end{tabular}

${ }^{a} \mathrm{C}$, cytoplasmic; $\mathrm{N}$, nuclear.

b The score used is explained in Materials and Methods. Numbers on the left and right correspond to Intensity and Proportion scores, respectively. The scores represent the average of 6 animals in each group.

Table 3 Hsp25-positive vessels in lymphomas

\begin{tabular}{lccc}
\hline Untreated & Doxorubicin & Lovastatin & $\begin{array}{c}\text { Lovastatin }+ \\
\text { doxorubicin }\end{array}$ \\
\hline $18.8 \pm 1.1$ & $14.9 \pm 2.2$ & $16.24 \pm 1.7$ & $13.3 \pm 1.17$ \\
\hline
\end{tabular}

The score used is explained in Materials and Methods. Numbers are mean \pm SE. Doxorubicin treatment significantly decreased $(P<$ $0.05)$, the number of Hsp25 vessels and more significantly when associated with lovastatin $(P<0.01)$, in both cases when compared with the control group. The scores represent the average of 6 animals in each group. In lymphomas, factors VIII and FXIIla did not reveal the endothelium of the blood vessels as did Hsp25.

of them had strong Hsp25 expression (Fig 4G). Isolated apoptotic cells between the viable tumor cells were Hsp25 negative. In DOX-treated lymphomas (obtained 3 or 11 days after treatment), only blood vessels showed Hsp25 expression (Fig 4F), and the number of Hsp25-positive vessels changed with the different treatments (Table 3).

LOV-treated breast carcinomas showed characteristics similar to those observed in DOX-treated group. In LOVtreated sarcomas, viable tumor cells showed higher Hsp25 expression than did controls because of the presence of weak but noticeable Hsp25 expression in the nuclear compartment. Massive apoptosis was Hsp25 negative or very weakly positive. The tumor cells surrounding the areas of massive apoptosis were Hsp25 positive in areas of cells changing their appearance to more elongated form. In these areas, Hsp25 appeared in the cytoplasm and, sometimes, in the nuclei of tumor cells. Individual apoptosis lacked Hsp25. LOV-treated lymphomas (obtained 3 or 11 days after treatment) lacked Hsp25 in viable tumor cells as well as in massive and individual apoptoses, but the protein remained in endothelial cells of blood vessels (Table 3).

In LOV- and DOX-treated breast carcinomas, viable tumor cells showed very low levels of Hsp25 expression (Table 2). Massive apoptosis displayed weak immunostaining for Hsp25, and periapoptotic cells were occasionally positive in the cytoplasm and the nucleus. Most of the individual apoptosis was Hsp25 negative. In LOVand DOX-treated sarcomas, viable tumor cells had more Hsp25 than did controls (Table 2). Figure 5 shows the total Hsp25 content in the tumors. Massive apoptosis areas were Hsp25 negative, but the tumor cells surrounding the areas of massive apoptosis had Hsp25 expression in certain areas, mainly those around the vascular tracts (Fig $4 \mathrm{H})$ and in cells changing their appearance to more elongated form. Individual apoptosis was Hsp25 negative. LOV- and DOX-treated lymphomas (obtained 3 or 11 days after treatment) lacked Hsp25 in viable tumor cells and in massive and individual apoptoses, and the protein remained in endothelial cells of blood vessels but at lower levels than in the controls (Table 3).

\section{Heat shock protein 70}

In untreated breast carcinomas, viable tumor cells expressed Hsp70 in the cytoplasm and nucleus (Table 4). In areas of massive apoptosis, the protein was positive in apoptotic cells, displaying a lesser degree of damage, and it was absent in areas with more advanced deterioration (cells displaying the corpses without the compact nuclear fragments). Sometimes, groups of tumor cells located in
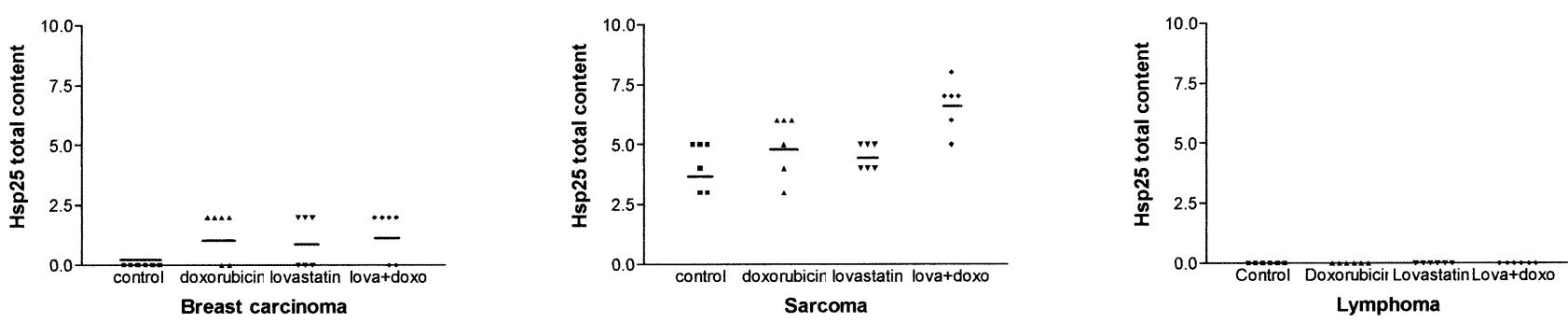

Fig 5. Hsp25 expression levels in viable tumor cells in untreated tumors and in tumors treated with doxorubicin (DOX) and lovastatin (LOV). The values represent the sum of the Hsp25 content in the cytoplasmic and nuclear cell compartments (using the evaluation scores explained in Materials and Methods). Note that sarcomas had the higher Hsp25 expression levels and that the levels changed after drug administration (Hsp25 was significantly higher $[P<0.001]$ in the group treated with LOV and DOX than in the control group). 
Table 4 Viable tumor cells, Hsp70 expression in untreated tumors and in tumors treated with doxorubicin (DOX) and lovastatin (LOV)

\begin{tabular}{|c|c|c|c|c|c|c|c|c|}
\hline \multirow[b]{2}{*}{ Tumor } & \multicolumn{2}{|c|}{ Untreated } & \multicolumn{2}{|c|}{ DOX } & \multicolumn{2}{|c|}{ LOV } & \multicolumn{2}{|c|}{ LOV + DOX } \\
\hline & $\mathbf{C a}^{\mathrm{a}}$ & $\mathbf{N}$ & C & $\mathbf{N}$ & C & $\mathbf{N}$ & C & $\mathbf{N}$ \\
\hline Breast carcinoma & $2+1^{b}$ & $2+2$ & $2+1$ & $1+2$ & $1+2$ & $1+2$ & $2+2$ & $1+2$ \\
\hline Sarcoma & $1+2$ & $1+2$ & $2+2$ & $1+2$ & $1+1$ & $2+3$ & $2+4$ & $2+4$ \\
\hline Lymphoma & $1+2$ & $1+2$ & $2+2$ & $1+2$ & $1+2$ & $1+2$ & $2+2$ & $1+2$ \\
\hline
\end{tabular}

${ }^{a} \mathrm{C}$, cytoplasmic; $\mathrm{N}$, nuclear.

b The score used is explained in Materials and Methods. Numbers on the left and right correspond to Intensity and Proportion scores, respectively. The scores represent the average of 6 animals in each group.
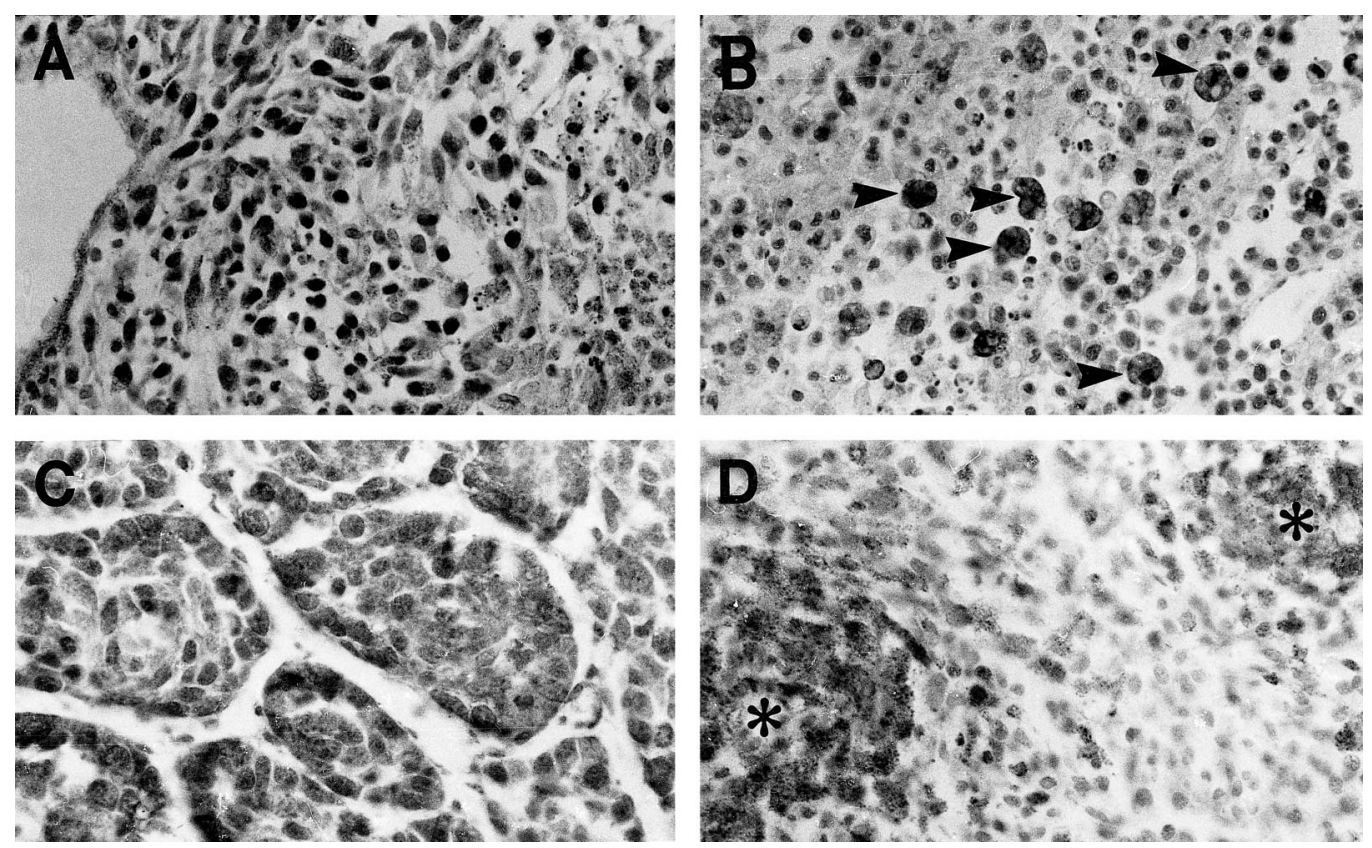

Fig 6. Hsp70 expression in spontaneous rodent tumors (immunostaining for Hsp70 with hematoxylin counterstaining). (A) Untreated sarcoma. Hsp70 can be seen in the nuclei of many viable tumor cells, whereas massive apoptotic cells (right side) lacked the protein. (B) Untreated sarcoma. Several macrophages showed Hsp70 in the cytoplasm; they were intermingled with small, round, viable sarcoma tumor cells and apoptotic cells. (C) Breast cancer treated with lovastatin (LOV) and doxorubicin (DOX). Hsp70 can be observed in the nuclei and cytoplasm of breast cancer cells that escaped apoptosis after chemotherapy. (D) Sarcoma treated with LOV and DOX. Viable tumor cells located around blood vessels $\left(^{*}\right)$ showed strong nuclear and cytoplasmic expressions of Hsp70.

the immediate vicinity of massive apoptosis showed Hsp70 in the nuclei and cytoplasm; however, most of the viable tumor cells close to massive apoptosis lacked Hsp70. Moreover, most of the isolated apoptotic cells lacked Hsp70. In untreated sarcomas, Hsp70 was observed in the cytoplasm and nucleus of viable tumor cells at similar levels than that observed in breast carcinomas (Table 4). In areas of massive apoptosis, the most important observation was the increase in Hsp70 content in the nuclei of cells in the vicinity of apoptosis (Fig 6A). Apoptotic cells, both isolated and in areas of massive apoptosis, were inconstant regarding Hsp70 expression. Most of them lacked the protein; however, apoptotic corpses had Hsp70 expression. Sometimes, large cells with features of phagocytosis showed Hsp70 expression (Fig 6B). In untreated lymphomas, viable tumor cells expressed Hsp70 (Table 4). In contrast, apoptotic cells lacked the protein.
In DOX-treated breast carcinomas, Hsp70 could be detected in the nucleus and cytoplasm of viable tumor cells (Table 4). Figure 7 shows the total Hsp70 content in the viable tumor cells. Hsp70 was inconstantly observed in cells in the areas of massive apoptosis and in individual apoptotic cells. In DOX-treated sarcomas, Hsp70-positive cells tended to be arranged surrounding blood vessels, and the total Hsp70 content increased with respect to control tumors (Fig 7). Massive apoptosis and individual apoptosis were in general Hsp70 negative. DOX-treated lymphomas (obtained 3 or 11 days after treatment) showed Hsp70 expression in viable tumor cells (Table 4). Massive and individual apoptoses did not show Hsp70 expression.

In LOV-treated breast carcinomas, Hsp70 expression was detected in viable tumor cells, mainly in the nuclei (Table 4). Hsp70 was inconstantly observed in cells in ar- 

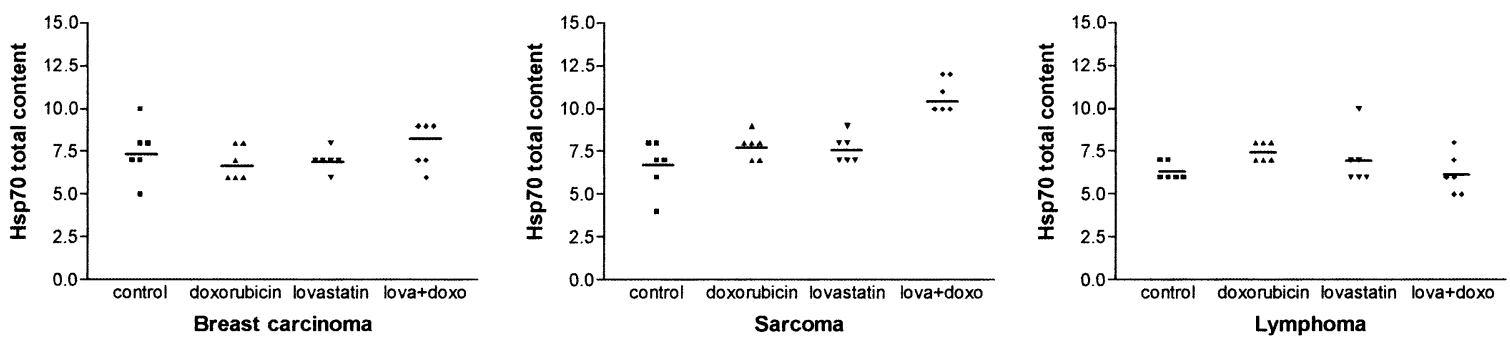

Fig 7. Hsp70 expression levels in viable tumor cells in untreated tumors and in tumors treated with doxorubicin (DOX) and lovastatin (LOV). The values represent the sum of the Hsp70 content in the cytoplasmic and nuclear cell compartments (using the evaluation scores explained in Materials and Methods). In sarcomas, note the increase in Hsp70 expression in doxorubicin-treated tumors and mainly in LOV- and DOXtreated tumors with respect to control tumors $(P<0.05$ and $P<0.001$, respectively).

eas of massive apoptosis and in individual apoptotic cells. In LOV-treated sarcomas, Hsp70-positive cells tended to be arranged surrounding blood vessels. Massive apoptosis and individual apoptosis were in general Hsp70 negative or weakly positive. LOV-treated lymphomas (obtained 3 or 11 days after treatment) showed Hsp70 expression in viable tumor cells (Table 4). Massive and individual apoptoses did not show significant Hsp70 expression.

In LOV- and DOX-treated breast carcinomas, Hsp70 expression in viable tumor cells was seen in cell clusters (Fig 6C). The total content of this protein showed a tendency to increase with respect to the other groups but without reaching statistical significance (Fig 7). Hsp70 was inconstantly observed in cells in the areas of massive apoptosis and in individual apoptotic cells. In LOV- and DOX-treated sarcomas, Hsp70-positive cells showed a predominance to be located surrounding blood vessels (Fig 6D). Total Hsp70 content increased with respect to the other groups (Fig 7). Massive apoptosis and individual apoptosis were in general Hsp70 negative. LOV- and DOX-treated lymphomas (obtained 3 or 11 days after treatment) showed Hsp70 expression in viable tumor cells (Table 4). Massive and individual apoptoses did not show significant Hsp70 expression.

Because the antibody against Hsp70 BRM22 used in the present study recognizes both Hsc70 and Hsp70, a specific antibody against Hsc70 was also tested in the tumor samples. The immunostaining of serial tumor sections with both antibodies showed that Hsc70 represented about $35-55 \%$ of the total Hsp70 content revealed by the BRM22 antibody.

\section{DISCUSSION}

Resistance to cytotoxic chemotherapeutic drugs is a major problem in the treatment of several types of cancers, and Hsps have been implicated among the molecular mechanisms of drug resistance. In this study, we found that the sarcoma was the tumor type that was more resistant to cell death, and this was noted in sarcomas growing both under control conditions and after cytotoxic drug administration. Sarcomas showed the highest expression levels of Hsp25 in the viable tumor cells growing under untreated conditions, and these levels increased after drug administration. This result is consistent with the concept that Hsp25 is implicated in survival of tumor cells (Ciocca et al 1992; Vargas-Roig et al 1997; Hansen et al 1999). Hsp25 has been found associated with enzymes of the base excision repair mechanism in HeLa cells (Mendez et al 2000) and involved in resistance to apoptosis (Samali et al 2001; Arrigo et al 2002). We also found that the expression levels of Hsp70 were similar in the 3 tumor types under control conditions but, after chemotherapy administration, only sarcoma tumor cells showed a significant increase in Hsp70 (of which a considerable amount, about $35-55 \%$, was Hsc70). In other words, sarcomas were the tumors with lower cell death, which displayed a competent Hsp70-Hsc70 and Hsp25 response, and had the highest levels of Hsp25 expression. The resistance of sarcomas to cell death after chemotherapy is in agreement with clinical situations; these tumors are usually more resistant to chemotherapy than are lymphomas and breast carcinomas.

In the present study, we found, in the 3 different tumor types, that cancer cells were dying by classic apoptosis. Massive apoptosis was the main phenomenon observed. However, apoptosis in isolated tumor cells (more significantly in lymphomas) was also observed The spontaneous apoptosis observed in untreated tumors, which could be triggered by many factors (eg, mild ischemia), was notably increased by the cytotoxic drugs. In previous studies, it has been pointed out that all the anticancer drugs can induce apoptosis (Hickman 1996). LOV affects the formation of farnesyl residues, decreasing the posttranslational processing (prenylation) of several proteins (including the p21 ${ }^{\text {ras }}$ oncoprotein) (Rozados et al 2000), and this may decrease the survival signaling in favor of apoptosis (Hickman 1996; Du et al 1999). Then, LOV through this effect may synergistically act with DOX to increase cell killing as noted in our study. It is of interest that in breast carcinomas, Hsp25 was clearly observed in 
the tumor cells with spontaneous massive apoptosis. The content of this Hsp decreased in treated tumors, perhaps because of the accelerated cell death induced by the cytotoxic drugs (apoptotic cells showed more advanced damage). In the future, it will be of interest to explore in detail the kinetic of Hsp25 expression during apoptosis. Therefore, our study presents evidence that in certain tumor types, Hsp25, rather than protecting the cells, participates in apoptosis as a late molecular event, perhaps tagging or routing the damaged proteins (or both) (or degrading enzymes) to the proteosomes or lysosomes cellular degrading systems.

Our detailed microscopic analysis revealed that many viable tumor cells close to apoptosis presented a translocation of Hsp25 and Hsp70 proteins to the nuclei. In this cellular location, there are important proteins that are in need of chaperones for maintaining their proper folding and for guiding their movements and interactions with deoxyribonucleic acid. This translocation was mainly observed in sarcomas.

This study revealed that not all viable tumor cells close to apoptosis expressed the Hsps, indicating that massive apoptosis is not affecting Hsp25 or Hsp70 expression in neighboring cells. This observation was more obvious in lymphomas and breast carcinomas. We also found that in breast carcinomas and lymphomas, there were tumor cells that escaped the action of the cytotoxic drugs and that did not express Hsp25 while Hsp70 levels remained stable. This finding suggests that other molecular mechanisms are protecting these cells from death. There are many molecular mechanisms that protect the tumor cells from chemotherapy (Bernal 1997). However, it is clear that in sarcomas, Hsp25 and Hsp70 are found in viable tumor cells located around the blood vessels. Interestingly, these are the areas that showed the most resistant tumor cell phenotype after chemotherapy. Finally, we found in lymphomas a unique feature: the expression of Hsp25 in endothelial cells, which was affected by the treatment with LOV and DOX. At present, we do not know the biologic significance of Hsp25 in endothelial cells of lymphomas. In conclusion, our study shows that each tumor type has unique features regarding the expression of Hsp25 and Hsp70 and that these proteins seem to be implicated in drug resistance mainly in sarcomas, whereas in breast carcinomas, Hsp25, rather than protecting the cells, could have a role in apoptosis as a late molecular event.

\section{ACKNOWLEDGMENTS}

Financial support for this research was provided by the National Research Council (CONICET) of Argentina, the Argentine Foundation for Cancer Research, and by the National University of Rosario, Argentina.

\section{REFERENCES}

Arrigo A-P, Paul C, Duchase C, et al. 2002. Small stress proteins: novel negative modulators of apoptosis induced independently of reactive oxygen species. Prog Mol Subcell Biol 28: 185-204.

Bernal SD (ed). 1997. Drug Resistance in Oncology. Marcel Dekker, Inc., New York.

Bonfil RD, Gonzalez AD, Siguelboim D, et al. 2001. Immunohistochemical analysis of Ki-67, p21 waf1/cip1 and apoptosis in marker lesions from patients with superficial bladder tumours treated with vinorelbine intravesical therapy in a preliminary phase I trial. BJU Int 88: 425-431.

Calderari S, Font MT, Garrocq O, Martinez S, Morini JC, Puche R, Tarrés MC. 1991. The inbred IIM/Fm stock. Rat News Lett 25: 28-29.

Celoria GC, Hinrichsen LI, Font MT. 1986. Tumor behavior of lymphoma TACB in rats resistant or susceptible to Sarcoma E-100. Comp Biol (Buenos Aires) 5: 73-83.

Ciocca DR, Clark GM, Tandon AK, Fuqua SAW, Welch MJ, McGuire WL. 1993. Heat shock protein hsp70 in patients with axillary lymph node-negative breast cancer: prognostic implications. J Natl Cancer Inst 85: 570-574.

Ciocca DR, Fuqua SAW, Lock-Lim S, Toft DO, Welch WJ, McGuire WL. 1992. Response of human breast cancer cells to heat shock and chemotherapeutic drugs. Cancer Res 52: 3648-3654.

Ciocca DR, Green S, Elledge RM, et al. 1998. Heat shock proteins hsp27 and hsp70: lack of correlation with response to tamoxifen and clinical course of disease in estrogen receptor-positive metastatic breast cancer (A Southwest Oncology Group Study). Clin Cancer Res 5: 1263-1266.

Ciocca DR, Puy LA, Fasoli LC. 1989. Study of estrogen receptor, progesterone receptor, and the estrogen-regulated $\mathrm{Mr} 24,000$ protein in patients with carcinoma of the endometrium and cervix. Cancer Res 49: 4298-4304.

Ciocca DR, Vargas-Roig LM. 1997. Heat shock proteins and drug resistance in breast cancer. In: Drug Resistance in Oncology, ed Bernal SD. Marcel Dekker, New York, 167-190.

Ciocca DR, Vargas-Roig LM. 2002. Hsp27 as prognostic and predictive factor in cancer. Prog Mol Subcell Biol 28: 205-218.

Cuello-Carrión FD, Ciocca DR. 1999. Improved detection of apoptotic cells using a modified in situ TUNEL technique. $J$ Histochem Cytochem 47: 837-839.

Di Masso RJ, Abdala S, Sanchez SM, Font MT. 1991. Respuesta a la selección divergente por conformación corporal en el ratón. Mendeliana 9: 79-92.

Doménico AD, Rabasa SL, Font MT, Suárez JM. 1963. Sarcoma E100. Ciencia e Investigación 19: 462.

Du W, Liu A, Prendergast GC. 1999. Activation of the PI3'K-AKT pathway mask the proapoptotic effects of farnesyltransferase inhibitors. Cancer Res 59: 4208-4212.

Hansen RK, Parra I, Lemieux P, Oesterreich S, Hilsenbeck SG, Fuqua SA. 1999. Hsp27 overexpression inhibits doxorubicin-induced apoptosis in human breast cancer cells. Breast Cancer Res Treat 56: 187-196.

Hickman JA. 1996. Apoptosis and chemotherapy resistance. Eur J Cancer 32A: 921-926.

Leist M, Jäättelä M. 2001. Four deaths and a funeral: from caspases to alternative mechanisms. Nat Rev 2: 589-598.

Matar P, Rozados VR, Binda MM, Roggero EA, Bonfil RD, Scharovsky OG. 1999. Inhibitory effect of lovastatin on spontaneous metastases derived from a rat lymphoma. Clin Exp Metastasis 17: 19-25.

Matar P, Rozados VR, Roggero EA, Scharovsky OG. 1998. Lovastatin 
inhibits tumor growth and metastasis development of a rat fibrosarcoma. Cancer Biother Radiopharm 13: 387-393.

Mendez F, Sandigursky M, Franklin WA, Kenny MK, Kureekattil R, Bases R. 2000. Heat-shock proteins associated with base excision repair enzymes in HeLa cells. Radiat Res 153: 186-195.

Nylandsted J, Rohde M, Brand K, Bastholm L, Elling F, Jaattela M. 2000. Selective depletion of heat shock protein 70 (Hsp70) activates a tumor-specific death program that is independent of caspases and bypasses Bcl-2. Proc Natl Acad Sci U S A 97: 78717876.

Puy LA, Lo Castro G, Olcese JE, Lotfi HO, Brandi HR, Ciocca DR. 1989. Analysis of a 24-kilodalton (KD) protein in the human uterine cervix during abnormal growth. Cancer 64: 1067-1073.

Rozados VR, Binda MM, Matar P, Gervasoni SI, Bonfil RD, Scharovsky OG. 2000. Inhibition of p21 ${ }^{\text {ras }}$ membrane anchorage by Lovastatin treatment of a rat lymphoma. Biocell 24: 171.

Samali A, Robertson JD, Peterson E, et al. 2001. Hsp27 protects mi- tochondria of thermotolerant cells against apoptotic stimuli. Cell Stress Chaperones 6: 49-58.

Scharovsky OG, Rozados VR, Gervasoni SI, Matar P. 2000. Inhibition of ras oncogene: a novel approach to antineoplastic therapy. $J$ Biomed Sci 7: 292-298.

Squartini F. 1979. Tumours of the mammary gland. In: Pathology of Tumors in Laboratory Animals. Volume III. Tumours of the mouse, ed Turusov VS. IARC Scientific Publications No 23, Lyon, France, 43-90.

Vargas-Roig LM, Fanelli MA, López LA, Gago FE, Tello O, Aznar JC, Ciocca DR. 1997. Heat shock proteins and cell proliferation in human breast cancer biopsy samples. Cancer Detect Prev 21: 441-451.

Vargas-Roig LM, Gago FE, Tello O, Aznar JC, Ciocca DR. 1998. Heat shock protein expression and drug resistance in breast cancer patients treated with induction chemotherapy. Int J Cancer (Pred Oncol) 79: 468-475. 